\title{
Der Roman Barlaam und Joasaph in seinem Verhältnis zu Agapets Königsspiegel.
}

Die Erzählung von Barlaam und Joasaph enthält an zwei Stellen einen kurzgefafsten Fürstenspiegel: sie schildert p. $308 \mathrm{ff}$. Boissonade in Joasaph das Ideal des Königs und legt Joasaph selbst p. 33। ff. eine Rede an Barachias über das richtige Verhalten des Herrschers in den Mund. Dafs beide Ausführungen sich grofsenteils wörtlich mit einer Anzahl Kapitel in Agapets $\sigma \chi \varepsilon \delta \delta \eta \beta \alpha \sigma \iota \iota x \eta ́$ decken, haben schon Damke ${ }^{1}$ ) und Boissonade ${ }^{2}$ ) erkannt, welche die meisten der in Frage kommenden Parallelen anführen. Noch nicht untersucht ist, welche Folgerungen aus dieser Übereinstimmung für das Verhältnis beider Werke zu einander zu ziehen sind. Boissonade, der die Frage, ob Johannes Damascenus als Verfasser des Mönchsromans anzusehen sei, als eine offene betrachtet ${ }^{3}$ ), spricht gleichwohl ohne weiteres Agapet die Priorität zu und hält dessen Königsspiegel für die Quelle der entsprechenden Abschnitte des Barlaam. ${ }^{4}$ ) Nachdem festgestellt ist, dafs Johannes Damascenus mit unserm Roman nichts zu thun hat, fehlt jeder äufsere Entscheidungsgrund, und das Verhältnis der beiderseitigen Darlegungen kann nur durch eine genaue Analyse klargelegt werden. Geboten ist eine solche Untersuchung vor allem durch die Frage nach Abfassungszeit und Quellen der Barlaamerzählung. Agapets Werk ist nach der Akrostichis unter Justinian verfalst.") Sollte sich ergeben, dafs es teil-

1) S. die Anmerkungen zu seiner Ausgabe d. Agapet (Basel 1633).

2) S. d. Ausgabe d. Barlaam (Anecdota Graeca IV), Paris 1832, p. 381² u. 8.

3) A. a. 0. praef. p. VII.

4) A. a. O. p. $331^{2}, 332^{2}, 333^{3}, 333^{5}$.

5) Ob unser Agapet der Rhetor ist, an welchen Prokop seinen 112. Brief richtete, steht dahin. Der stark rhetorisierende Charakter des Schriftchens (s. unten; vgl. auch Seitz, Die Schule von Gaza S. 14) scheint dafür zu sprechen. Wenn Seitz dagegen das chronologische Verhăltnis geltend macht, da A. in jenem Briefe als gereifter Mann und achtbarer Kritiker erscheine, so legt er wohl einigen Hoflichkeitsphrasen $z u$ viel Gewicht bei. Übrigens konnte A. sehr wohl gleichaltrig mit P. und sogar etwas älter als dieser sein, ohne dafs wir ihm deshalb die dem Justinian gewidmete Schrift absprechen müfsten. 
weise aus dem Mönchsromane geflossen ist, so müfste der letztere spätestens etwa fünfzig bis siebzig Jahre früher entstanden sein als Zotenberg ${ }^{1}$ ) annimmt. Im umgekehrten Falle gewännen wir aufser der Kenntnis einer Quelle des Barlaam einen interessanten Einblick in die Art, wie der Verfasser seine Quellen benutzte. Während seine Ausbeutung des Aristides ${ }^{2}$ ) eine sehr einfache und bequeme war, hätte der Autor sich ein anderes Mal.die Mühe genommen, aus Sätzen seiner Vorlage einen Cento zusammenzuweben und sich dieser Aufgabe mit anerkennenswertem Geschicke entledigt. Eine nur mittelbare Verwandtschaft zwischen beiden Werken endlich würde uns auf die Spur einer neuen bisher unentdeckten Quelle des Barlaamromans führen, deren Auffindung vielleicht dem Zufalle oder eifriger Nachforschung vorbehalten wäre.

Die beiden Ausführungen über das Fürstenideal in der Mönchserzählung bilden jede für sich ein wohlgeordnetes Gedankengefüge. Die nämlichen Sätze, die hier Glieder dieses organischen Ganzen sind, erscheinen bei Agapet oft weit von einander getrennt und entweder völlig isoliert oder mit fremden Gedanken zu einem Kapitel vereinigt. Was diese Kapitel selbst wieder unter einander verknüpft, ist das rein äufserliche Band der Akrostichis. Nur in seltenen Fällen entsprechen mehrere auf einander folgende Kapitel Agapets einer Gedankenreihe des Barlaam. Die Erörterung an der ersten Stelle des Romans (p. 308 ff.) nimmt folgenden Verlauf: Der zum Christentum bekehrte Joasaph geht als König allen in der Bekämpfung des alten Irrtums mit gutem Beispiele voran und erfüllt viele mit seiner Gesinnung, wie denn überhaupt die Unterthanen nach dem Bilde des Herrschers sich modeln. ${ }^{3}$ ) So wuchs unter den Leuten die Frömmigkeit, und der König hielt fest

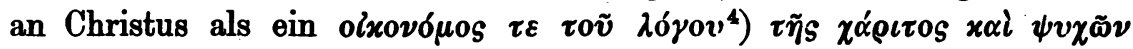

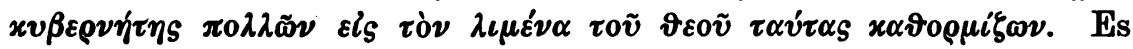

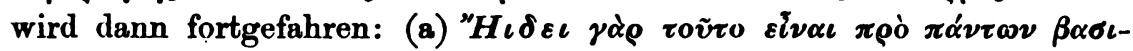

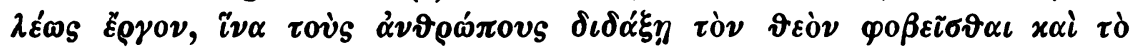

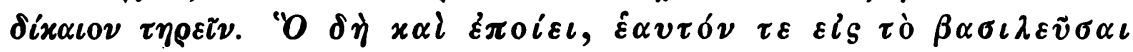

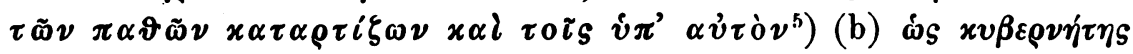

1) Notices et extraits 26,1 p. $51 \mathrm{ff}$. u. $100 \mathrm{ff}$.

2) Vgl. Texts and studies edited by J. Armitage Robinson I 1 p. $35 \mathrm{ff}$.

3) Zum Gedanken vgl. meine Bemerkungen Bd. I S. 410 dieser Zeitschrift

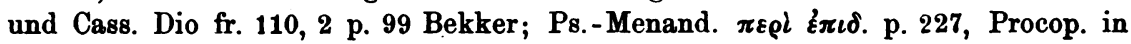
Anast. p. 43 Vill., Basil. Maced. exhort. c. 19.

4) Vgl. Basil. regul. brev. tract. p. 1112 b Migne, de fide 677 a.

5) Das in gesperrter Schrift Gedruckte hat bei Agapet keine Paralielo. 


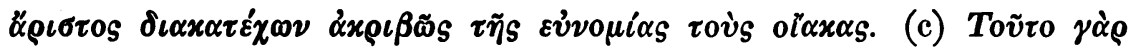

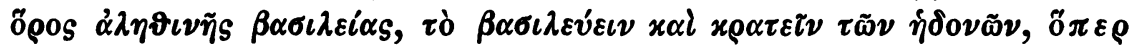

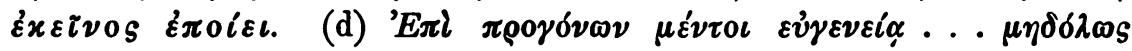

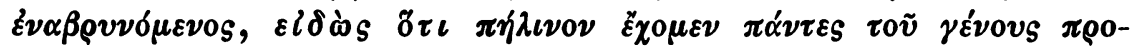

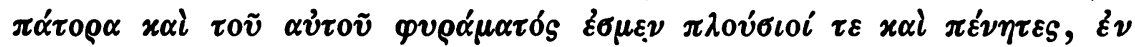

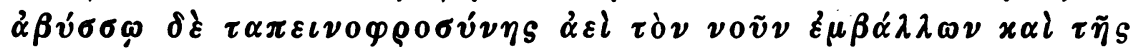

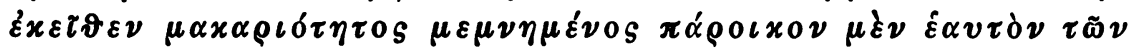
$\varepsilon \nu \tau \alpha \tilde{v} \vartheta \alpha \dot{\varepsilon} \lambda \circ \gamma \dot{\jmath} \xi \varepsilon \tau \ldots$

Nachdem er nun alle von ihrem Irrtum bekehrt hatte, heifst es weiter, fafste Joasaph ein zweites ins Auge, die Wohlthätigkeit. $\Sigma \omega \varphi \rho o-$

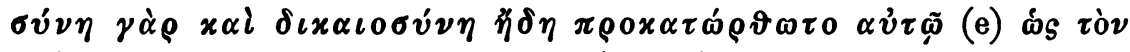

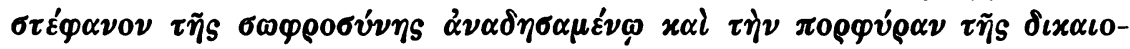

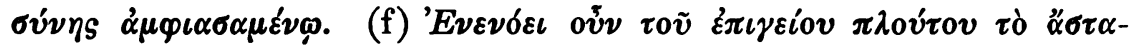

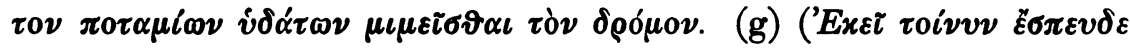

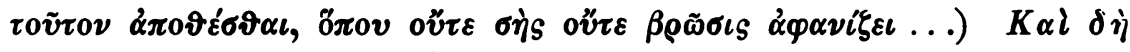

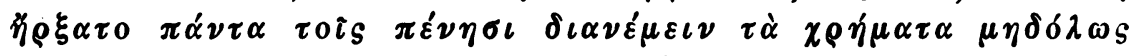

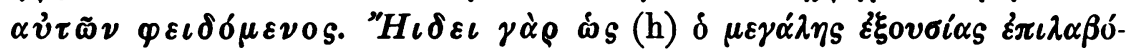

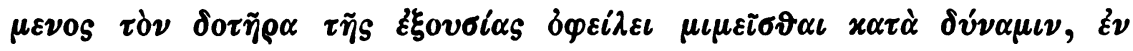

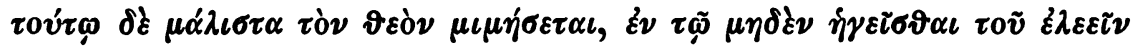

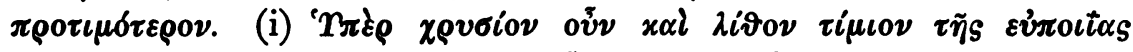

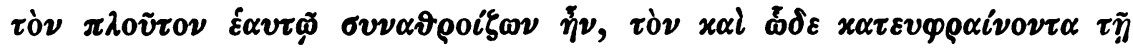

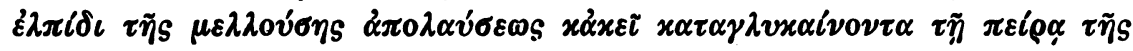

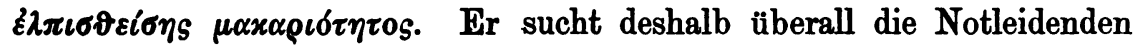

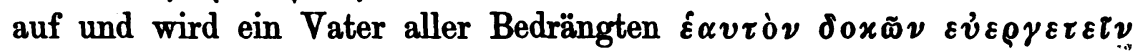

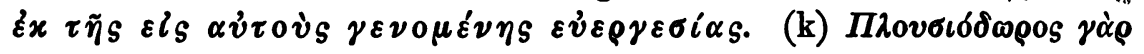

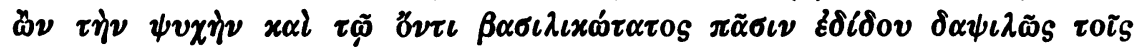

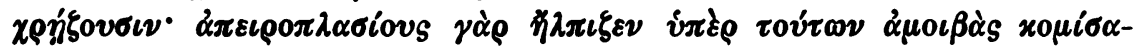

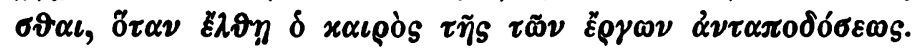

Der Zusammenhang läfst in diesem ganzen Abschnitt nichts zu wünschon übrig. Nachdom in a die Aufgabe des christlichen Königs klar formuliert ist, wird bei der weiteren Ausführung des Themas das zu Anfang angeschlagene Motiv von der Macht des Beispiels wieder aufgenommen: der König wirkt auf seine Unterthanen in erster Linie dadurch ein, dafs er sich selbst von aller Leidenschaft reinigt. Dies leitet über zu dem in Darstellungen des Fürstenideals sehr beliebten ${ }^{1}$ ) Gedanken: der wahre König zeigt sich in der Herrschaft über sich

1) S. Bd. I dieser Zeitschrift S. 403. Aus der kirchlichen Litteratur gehört u. a. St. hierher Basil. in Isai. prophet. p. 297 a f. Migne: 'O $\alpha \lambda \eta \vartheta \tilde{\sigma} s \alpha^{\prime} \rho \chi \omega \nu$ ov่u

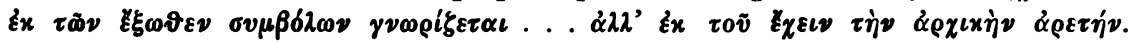

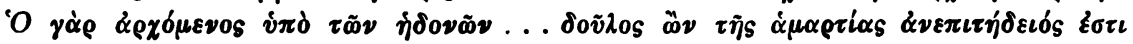

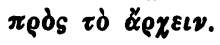


selbst, nicht in Äulserlichkeiten - hier der Abkunft und dem Ansehen,

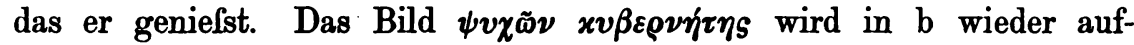
genommen. Nach Besprechung der Selbstbeherrschung und der Gerechtigkeit - es wird auf die vorhergehende Behandlung dieser Tugenden

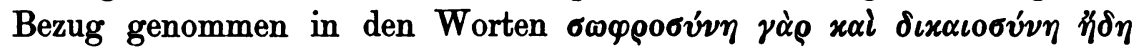

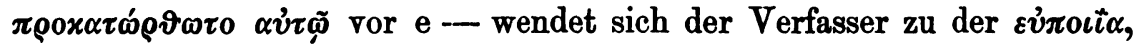
die hièr die in den antiken Königsspiegeln und Fürstenenkomien an zahlreichen Stellen betonte $\varphi\left(\lambda \alpha \nu \vartheta \rho \omega \pi i \alpha^{1}\right)$ vertritt $\left.{ }^{2}\right)$ und wie diese ${ }^{3}$ ) aus der Aufgabe des Fürsten, der Gottheit nachzuahmen, abgeleitet wird (h). Noch vorher wird das Gebot der Wohlthätigkeit begründet durch den Hinweis auf die Vergänglichkeit alles Irdischen und die Notwendigkeit, durch Wohlthun sich ein Kapital im Himmel zu sammeln (fg). Dieser Gedanke beherrscht den ganzen Schlufs des Abschnitts: J. erwirbt sich jenen Schatz, er geht überall den Mangelleidenden nach, denn er glaubt, dadurch sich selbst zu nützen und erwartet reiche Entschädigung am Tage der Vergeltung (ik).

Agapet bringt den Gedanken über die Aufgabe des Königs (a) im 1. Kapitel, diesmal in einer stark abweichenden Form, von der unten noch die Rede sein wird. b bildet mit einer Erweiterung A.s 2. Kapitel. Der Bemerkung e begegnen wir im 18., d im 4. Kapitel. e ist in Kap. 18 an $\mathrm{c}$ angeschlossen, $\mathrm{f}$ erscheint im Anfang von Kap. 7 wieder. $g$ entspricht dem Sinne nach Kap. 67 Satz $3 \mathrm{ff}$., doch ist die Übereinstimmung hier nur allgemeinerer Art, und es ist bei der ungemeinen Verbreitung des Gedankens ${ }^{4}$ ) die Möglichkeit vorhanden, dafs eine direkte Beziehung auch nur im Sinne der Benutzung einer und derselben Quelle nicht vorliegt. h bildet mit einer Erweiterung A.s 37. Kapitel, i schliefst sich, wie im Barlaam, so auch bei A. an (38), $\mathbf{k}$ endlich entspricht der 2. Hälfte von Kap. 44.

Das nämliche Verhältnis besteht zwischen Agapet und dem zweiten Abschnitte des Romans p. 331 ff. Joasaph, der sich in die Einsamkeit

1) Vgl. Xer. Cyrop. 8, 2,1; Themist. or. 1 p. $5 \mathrm{c}$ u. ö.; Theophyl. inst. reg. II c. 26 . Synes. de regn. c. 28 p. 29 a; s. auch Julian. fragm. ep. p. 372 Hertl.

2) $\varepsilon \dot{v} \pi 0$ t $\alpha$ in diesem Zusammenhange auch Themist. or. 15 p. $192 \mathrm{~b}$.

3) Ael. Aristid. or. 9 p. 107 Dind., Dio Chrys. or. 3 p. 53 Dind., Themist. or. 1 p. $8 \mathrm{c}$, or. 6 p. $78 \mathrm{~d}$, or. 11 p. $146 \mathrm{cf}$, or. 19 p. $226 \mathrm{~d}$. Vgl. auch die in der vor. Anmerk. angeführte Stelle u. Greg. Naz. or. 14 p. $276 \mathrm{~b}$.

4) Zu Grunde liegt Matth. VI $19 \mathrm{ff}$. Von den zahlreichen Stellen der patristischen Litteratur, die den Gedanken ausführen, wären (aufser hist. Barl. et Joas. p. 126, 128) etwa zu vergleichen Basil. de renunt. saec. p. 632 a Migne; hom. IX in hexaem. p. 193c; Gregor. Naz. or. 14 p. 269 c, 270 b der Par. Ausg. v. 1840. Innerhalb der heidnischen Litteratur ist verwandt Themist. or. 15 p. 193c: $\mu$ óv

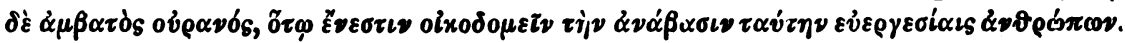


zurückzieht und statt seiner Barachias zum Könige ernennt, führt diesen mit folgenden Ermahnungen in sein Amt ein. Du hast schon vor mir, so beginnt er, Gott erkannt und ihm gedient; jetzt wende noch grölseren

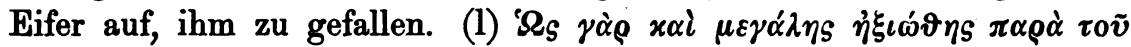

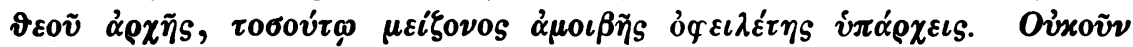

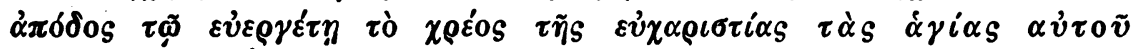

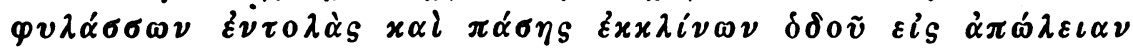

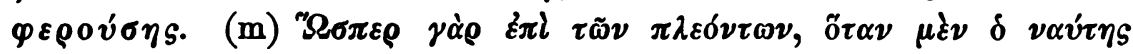

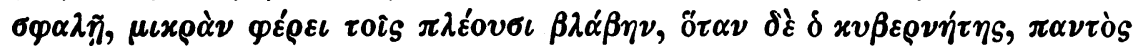

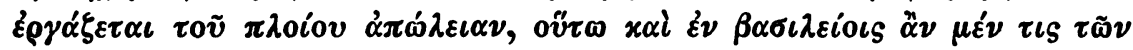

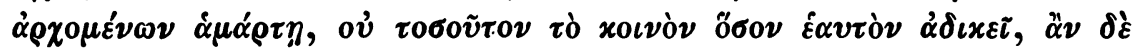

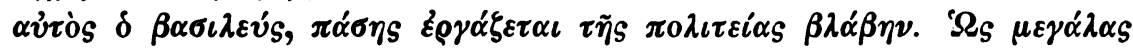

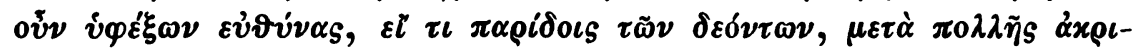

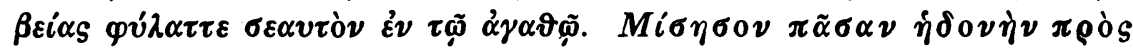

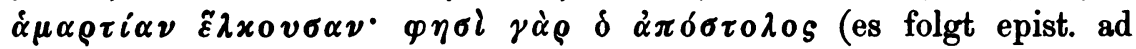

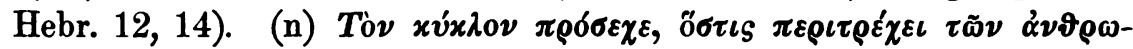

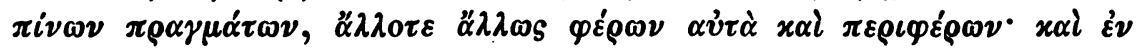

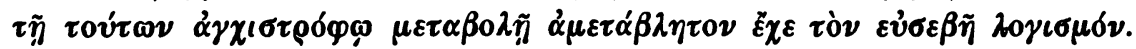

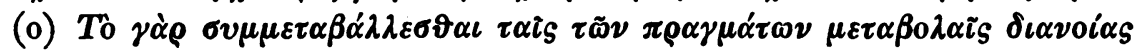

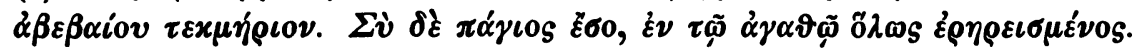

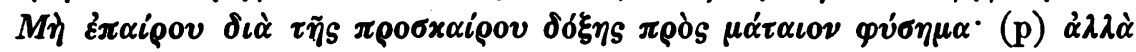

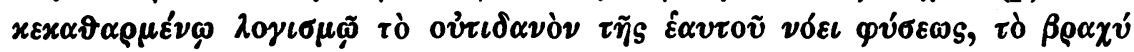

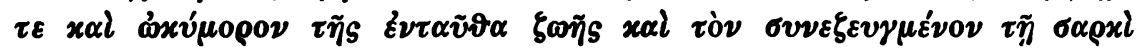

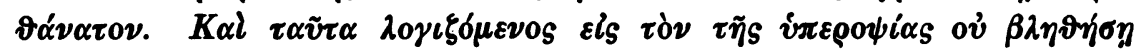

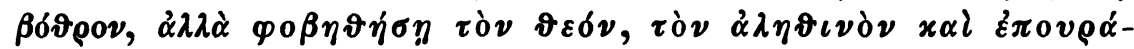

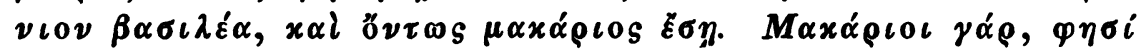

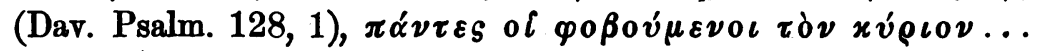

Nach dieser allgemeinen Paränese werden im besonderen B. drei Gebote eingeschärft: 1) Sei barmherzig. Diese Tugend wird vor allem

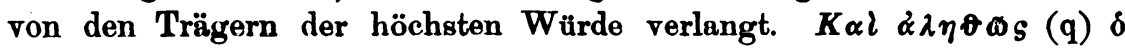

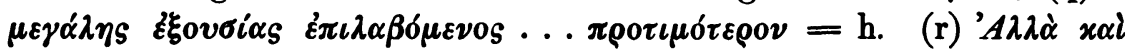

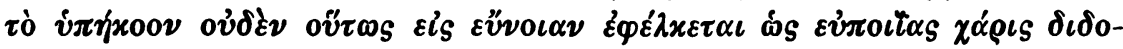

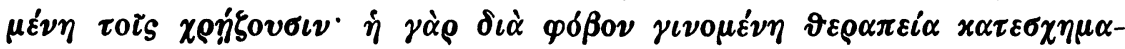

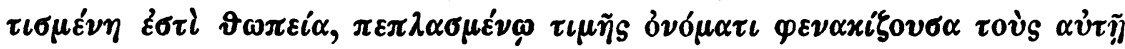

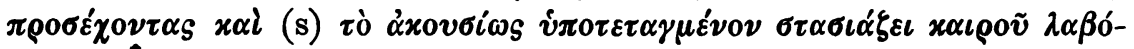

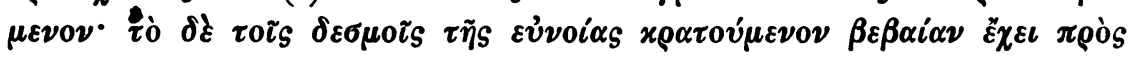

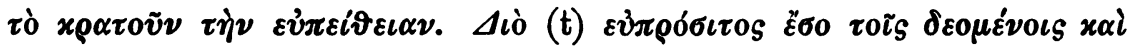

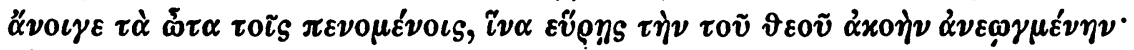

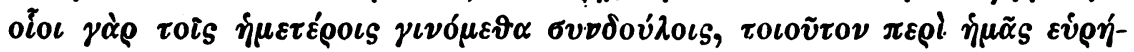

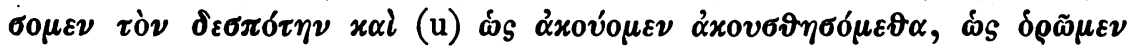

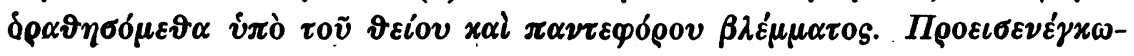




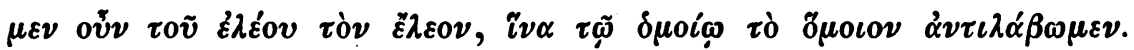
2) Vergieb, und dir wird vergeben werden. Daher trage kein Ver-

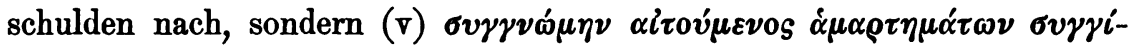

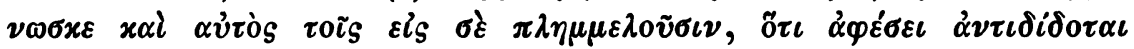

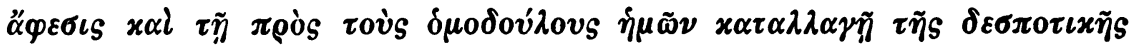

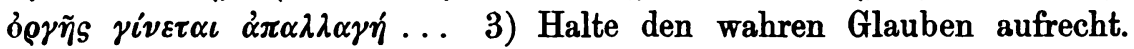
Dieser Passus hat keine Parallele bei Agapet.

Auch hier hängt mit einer Ausnahme alles sehr gut in sich zusammen: nur vor $n$ bricht der alte Faden ab, und ein neuer wird angesponnen. Der allgemeine Teil zerfällt so in zwei Abschnitte, deren erster den Gedanken ausführt: achte auf dich und sei dir deiner Verantwortung bewufst, während der zweite das Gebot enthält: lafs dich durch den Gedanken an die Vergänglichkeit alles Irdischen vor Überhebung bewahren. Daran fügt sich sehr passend als Abschlufs dieses Teiles das Versprechen der himmlischen Seligkeit. Für die Barmherzigkeit werden drei Motive geltend gemacht: die Pflicht, Gott nachzuahmen, die Sicherung der weltlichen Macht des Herrschers durch das Wohlwollen der Unterthanen und - mit Recht wieder an letzter Stelle die Barmherzigkeit Gottes am Tage des Gerichts. Die Ausführung über die Sündenvergebung ist durchaus einheitlich und behandelt nur den einen Gedanken: Vergieb und dir wird vergeben.

Bei Agapet sind die einzelnen Teile dieses Gefüges grolsenteils wieder weit von einander getrennt. Nur $m$ und $n$ stehen auch bei ihm als Kap. 10 und 11 bei einander, ebenso o und $p$ als Kap. 13 und 14. Zwischen diesen beiden Kapitelpaaren steht als Kap. 12 eine Erörterung über Schmeichler und die von ihnen dem Fürsten drohende Gefahr. 1 bildet den Anfang von Kap. 5, q mit einer Erweiterung Kap. 37, r macht den gröfsten Teil von $19, \mathrm{~s}$ von 35 aus, $t$ entspricht Kap. 8, u Kap. 23, $v$ endlich begegnet uns in Kap. 64 wieder. ${ }^{1}$ )

Dieser Sachverhalt ist nun schon der Annahme, dafs der Verfasser des Barlaam aus Agapet geschöpft habe, recht ungünstig. Wer nach bunten Steinchen sucht, kann durch Zertrümmerung eines Mosaikbildes mit leichter Mühe sein Ziel erreichen. Ein besonders glücklicher Zufall aber müfste obwalten, wenn sich eine Anzahl planlos zusammengetragener Steinchen zu einem wohlgefügten Mosaikbilde sollte ordnen lassen. Man vergleiche besonders $m-p$ mit den entsprechenden Kap. 10-14 bei A.; hier drängt sich mit Gewalt die Annahme auf, dafs der Zusammen-

1) Noch vollständiger ist der Zusammenhang zerrissen im cod. Vindob. phil. graec. 167 saec. XV-XVI, den ich zu vergleichen Gelegenheit hatte. Kap. 10 behält hier seine Stelle, Kap. 11 dagegen tritt an den Platz des 68., das 13. wird zum 66., das 14. zum 19. Kap. 
hang, wie ihn der Roman bietet, das Ursprünglichere ist, und dafs A. das ganz heterogene Kapitel über die Schmeichler eingeschoben und

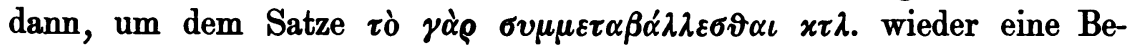

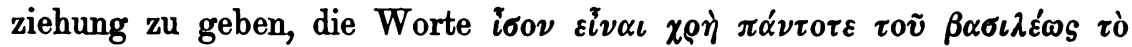

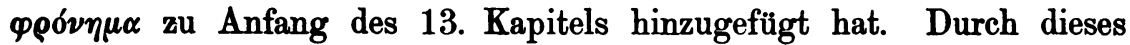
Mittel ist hier die Wunde gut geheilt. Anderwärts sind Narben zurückgeblieben, welche an die Zerreilsung des Zusammenhanges erinnern. Nachdem s (c. 35) von r (c. 19) getrennt worden war, erhielt der Satz

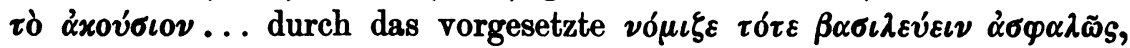

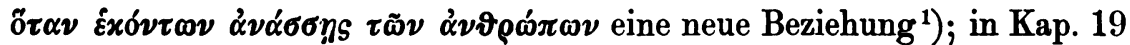

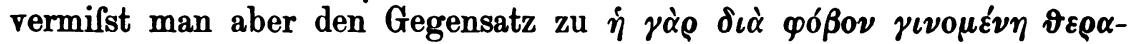

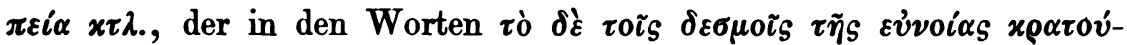

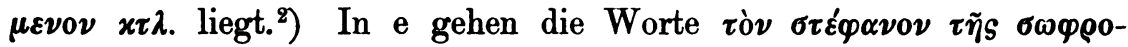

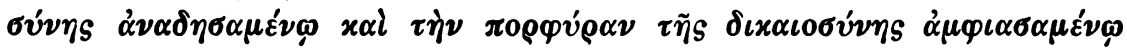

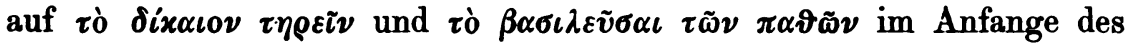
ganzen Abschnittes; Agapet bezieht sie in c. 18 nur auf das letztere

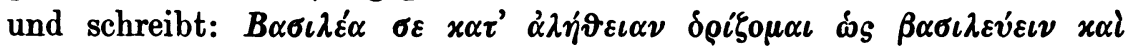

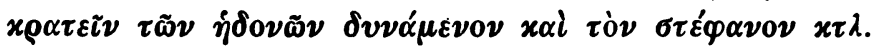

Man beachte ferner, wie im Barlaam das Stück über die Vergebung ( $v$ und das ihm Vorangehende) aus der Stelle Matth. 6, 15 (vgl. Marc.

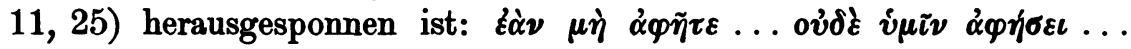

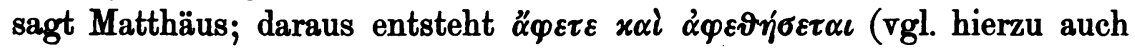

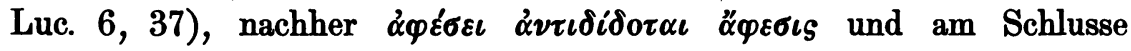

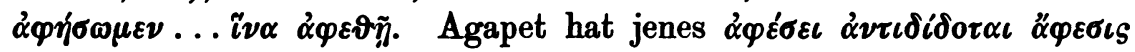
gleichfalls, aber ohne es auf die Bibelstelle zu gründen. ${ }^{3}$ )

1) Der Gedanke, dafs nur ein wohlwollendes Regiment die Herrschaft sichere, wahrend die Gewalt sie gefährde, ist in der antiken Litteratur sehr häufig. Unter den KS. bringt ihn Gregor von Nazianz or. 2 p. 19 df. unter Anwendung des Aus-

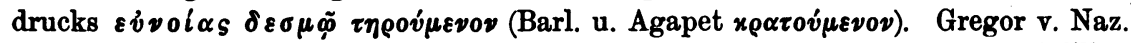
giebt nun allerdings wie Agapet 35 Anf. die Weisung, sich auf den guten Willen der Untergebenen, nicht auf Gewalt zu stützen; indes ist die Ubereinstimmung in diesem Satze zu allgemein, als dals darauf etwas zu geben wäre.

2) Allerdings enthalten die Sätze in ihrer Verbindung im Barl. einen logischen Fehler. In straffere Form gebracht lautet der Gedanke: Wohlwollen wird am besten hervorgerufen durch Wohlthun. Denn die Furcht schafft nur Heuchelei; aber das durch Wohlwollen Beherrschte leistet dauernden Gehorsam. Das Wohlwollen ist also zuerst Zweck, dann nur Mittel zum Zweck, während jetzt Zweck der Gehorsam ist. Doch darf man, glaube ich, einer solchen logischen Diskrepanz zwischen Anfang und Ende eines längeren Satzes nicht allzu viel Gewicht beilegen.

3) Es liefse sich auch darauf hinweisen, dafs d und das unmittelbar Folgende mit Greg. Naz. or. 33 p. 611 bf der Pariser Ausg. v. 1840 mehr Berührungen darbietet, als die entsprechende Stelle (c. 4) bei A. Allen gemeinsam ist der Gedanke: 
Höchst unwahrscheinlich ist es also, dafs die Fürstenspiegel des Barlaamromans Centone aus Agapet sind - aber nicht unmöglich. Wir wissen noch so wenig von der litterarischen Arbeitsweise jener Zeit, dafs Behutsamkeit geboten ist. Centone, wie sie Symeon Metaphrastes allerdings Jahrhunderte nach der Abfassung des Barlaam aus Basileios' des Grofsen Werken zusammenstellte, mahnen zur Vorsicht, und diese ist hier doppelt geboten, da wenigstens im ersten der beiden Abschnitte des Barlaam, wie später gezeigt werden soll, deutliche Spuren darauf hinweisen, dafs eine fremde Vorlage verarbeitet wurde.

Festere Anhaltspunkte zur Lösung unserer Frage bietet der rhetorische Charakter des agapetischen Werkes. Es sind die gewöhnlichen rhetorischen Kunstmittel, die A. fast Satz für Satz, oft in der geschraubtesten Weise, zur Anwendung bringt, vor allem Reime und Gleichklänge jeder Art und möglichst entsprechender Bau der Glieder einer Periode. $\mathrm{Zu}$ letzterem Zwecke wird die Zusammenziehung von Sätzen mit gemeinsamem Verbalbegriffe gern vermieden und statt dessen der Verbalbegriff an zweiter Stelle durch ein neues Verbum ausgedrückt, wodurch zugleich auch wieder häufig Gelegenheit zu Reimen (infolge Übereinstimmung der Verbalendungen) geboten wird. Ich wähle aus der Fülle der Beispiele

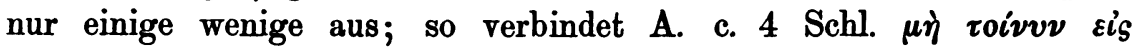

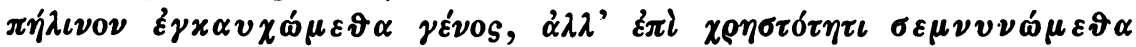

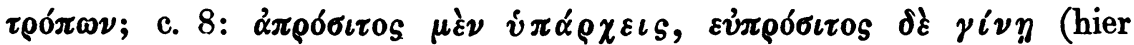
ohne Reim nur zum Zwecke genauer Korresponsion); c. 15: $\delta$ jò $\varrho$

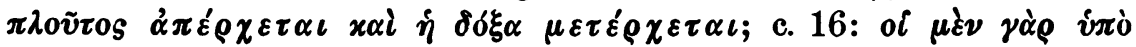

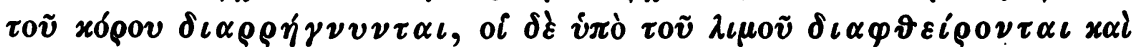

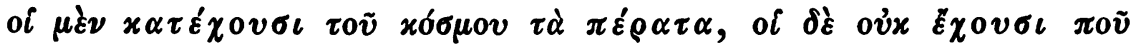
$\sigma \tau \tilde{\eta} \sigma \alpha \iota \tau \dot{\alpha} \pi \dot{\varepsilon} \lambda \mu \alpha \tau \alpha$; c. 29: $\ddot{\eta} \mu i \mu \eta \sigma \iota \nu \tau \tilde{\omega} \nu x \alpha \lambda \tilde{\omega} \nu \varepsilon^{\prime} x \delta \iota \delta \alpha^{\prime} \sigma x \varepsilon \tau \alpha \iota$ ทै $\mu \varepsilon i \omega \sigma \iota \nu \tau \tilde{\omega} \nu x \alpha x \tilde{\omega} \nu \varepsilon x \pi \alpha \iota \delta \varepsilon \dot{v} \varepsilon \tau \alpha \iota$.

Vergleicht man nun diejenigen Stellen beider Schriften mit einander, an welchen bei wörtlicher Übereinstimmung im ubrigen eine

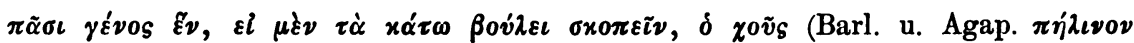

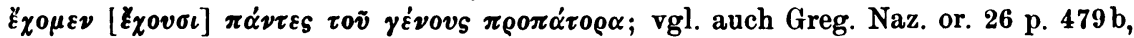
in divit. male mor. v. 22 (p. 540)). Gregor knüpft daran Erörterungen über wahre

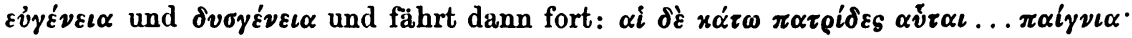

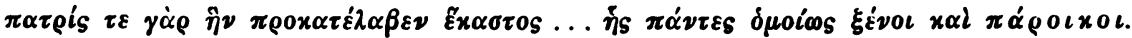

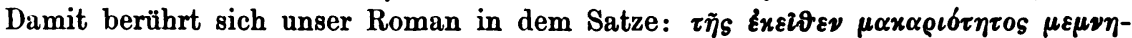

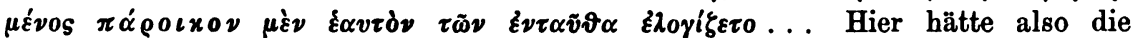
Barlaamerzählung mehr aus der Quelle als A. und könnte somit nicht durch dessen Vermittelung auf dieselbe zurückgehen. Indes handelt es sich um eine Utbereinstimmung in allgemeinen und weit verbreiteten Gedanken, nicht in charakteristischen Wendungen, und ich möchte daher nicht dafür einstehen, dafs der Passus wirklich aus der Stelle Gregors geflossen ist. 
Verschiedenheit in einzelnen Wendungen sich zeigt, so läfst sich fast überall leicht einsehen, wie A. bei seinem Haschen nach rhetorischen Effekten dazu kam, den in B. vorliegenden Wortlaut zu ändern, wäh- rend sich schwer ein Grund auffinden liefse, der den Verfasser des B. zur Änderung von A.s Text bewogen haben könnte. Man wird einwenden, der Autor des Romans habe durch solche Abweichungen den Sätzen seiner Quelle ihre rhetorische Manieriertheit abstreifen wollen. Zunächst ist ein derartiges Streben dem Verfasser fremd; er geht Gleich-

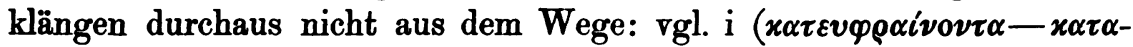

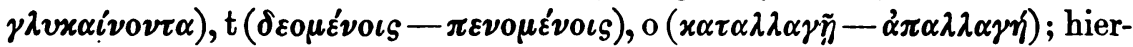

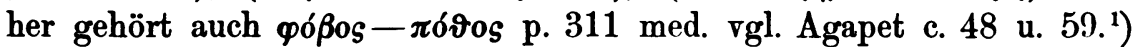
Ferner aber weicht der Wortlaut auch an solchen Stellen ab, an welchen ein Gleichklang im B. gar nicht entstehen würde, da er von den beiden entsprechenden Gliedern bei A. nur eines wiedergiebt. Ein gutes Beispiel hierfür bildet $f$ verglichen mit Agapet c. 7 :

\section{Barlaam:}

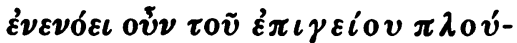
$\tau 0 v \tau$ cò $\alpha \sigma \tau \alpha \tau 0 \nu \pi 0 \tau \alpha \mu i \omega \nu$ v่ $\alpha^{\prime} \tau \omega \nu$ $\mu \iota \mu \varepsilon \tilde{\sigma} \sigma \alpha \iota \tau \grave{\nu} \nu \delta \rho \delta \mu \nu \nu$.
Agapet:

$\tau \tilde{\omega} \nu \dot{\varepsilon} \pi \iota \gamma \varepsilon \dot{i} \omega \nu \chi \rho \eta \mu \alpha \dot{\alpha} \boldsymbol{\tau} \nu \ddot{\alpha} \sigma \tau \alpha-$ $\tau 0 S \pi \lambda o \tilde{v} \tau 0 S \tau \tilde{\omega} \nu \pi 0 \tau \alpha \mu i \omega \nu \oint \varepsilon v-$

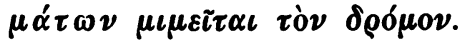

Hier ist $\boldsymbol{v} \delta \alpha ́ \tau \omega \nu$ durch $\oint \varepsilon v \mu \alpha ́ \tau \omega \nu$ ersetzt, weil sich so ein vollerer Reim zu $\chi \rho \eta \mu \alpha \dot{\tau} \tau \omega \nu$ ergiebt; die Verbindung $\dot{\varepsilon} \pi \iota \gamma . \chi \rho \eta \mu$. $\pi \lambda$. ist selbst wieder eben dieses Reimes wegen (auch $\dot{\varepsilon} \pi \iota \gamma \varepsilon i \omega \nu$ - $\pi 0 \tau \alpha \mu i \omega \nu$ kommt in Betracht)

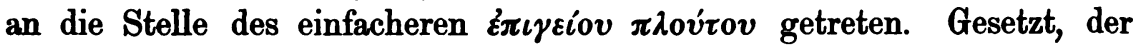
Verfasser des B. habe den Reim vermeiden wollen, was bewog ihn, nach der Ausmerzung von $\chi \rho \eta \mu \alpha ́ \tau \omega \nu$ nun noch $\rho \varepsilon v \mu \alpha ́ \tau \omega \nu$ mit $\dot{\delta} \delta \alpha ́ \tau \omega \nu$ zu vertauschen?

Einen ähnlichen Sachverhalt ergiebt folgende Gegenüberstellung:

\section{Barl. o-p.}

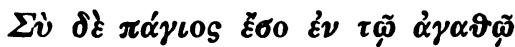

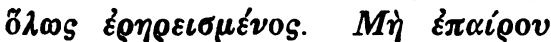

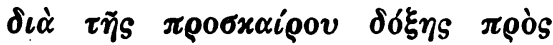
$\mu \alpha^{\prime} \tau \alpha \iota \nu \nu \varphi v ́ \sigma \eta \mu \alpha^{\cdot} \dot{\alpha} \lambda \lambda \dot{\alpha} x \varepsilon x \alpha \vartheta \alpha \rho \mu \varepsilon \dot{\nu} \omega$

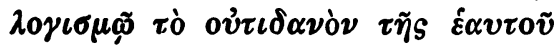

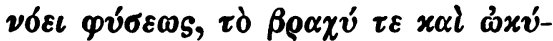

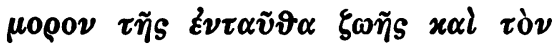

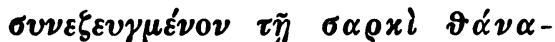

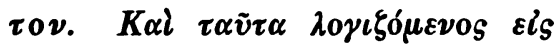

Agap. c. 13 u. 14.

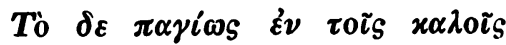

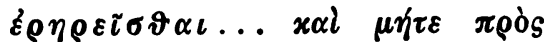

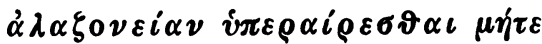

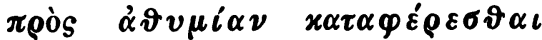

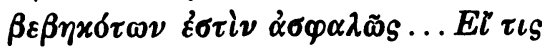

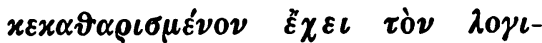

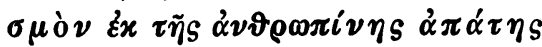

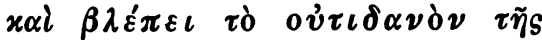

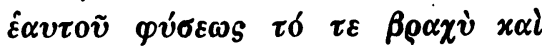

1) Derselbe Gegensatz bei Themist. or. 3 p. 48 b, aber abgeschwächt durch ein dazwischentretendes ępos. 
K. Praechter: Barlaam u. Joasaph im Verhältnis zu Agapets Konigsspiegel 453

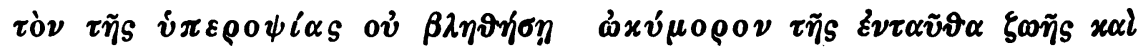

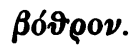

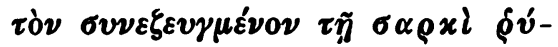

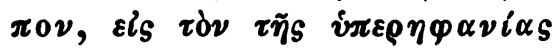

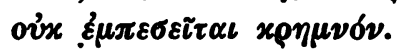

Bei Agapet haben rhetorische Rücksichten die Aufeinanderfolge von $\varepsilon^{z} \chi \varepsilon \iota$ und $\beta \lambda \varepsilon \dot{\pi} \varepsilon \iota$ hervorgerufen; die Veränderung der Konstruktion

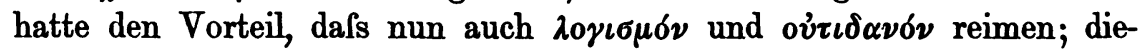

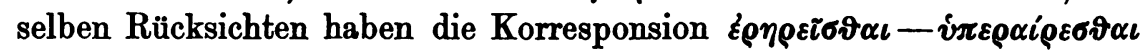
veranlafst und zur Hinzufügung eines dritten Gliedes $\mu \nmid \tau \varepsilon \varepsilon$. $\dot{\alpha} \vartheta$. $x \alpha \tau \alpha \varphi$. geführt, in welchem $\dot{\alpha} \vartheta v \mu i \alpha \nu$, wieder an $\dot{\alpha} \lambda \alpha \xi o \nu \varepsilon i \alpha \nu$ anklingt. Dieses neue Glied liefs sich erst einfügen, als der Zusammenhang von o und

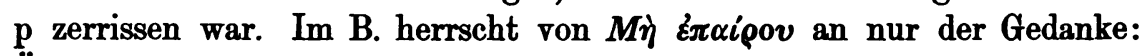
Überhebe dich nicht im Bewulstsein deiner königlichen Würde. Zur Warnung vor Niedergeschlagenheit im Unglück war hier kein Platz. Der Annahme aber, der Verfasser des Barl. habe, eben um einen $\mathrm{Zu}$ sammenhang herzustellen, das dritte Glied gestrichen, steht die zweite Hälfte des Abschnittes entgegen, die wieder deutlich zeigt, dals der Rhetor der Ändernde war. Der rhetorische Gesichtspunkt, der hier

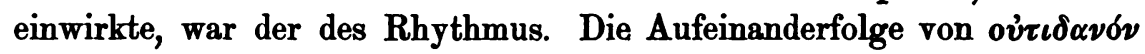

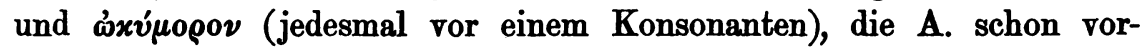
fand, mag ihn darauf gebracht haben, dem Choriambus in dem ganzen Kapitel eine hervorragende, dem Ohre leicht bemerkbare Rolle zu

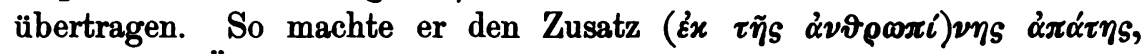

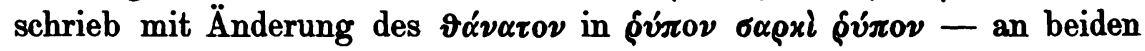
Orten geht der Rhythmus durch seine Stelle im Satze sehr stark zu

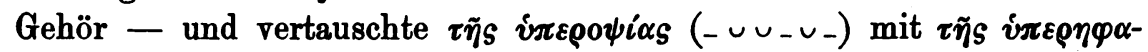
vías (-vv-uv-). ${ }^{1}$ )

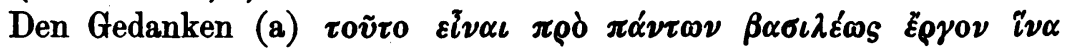

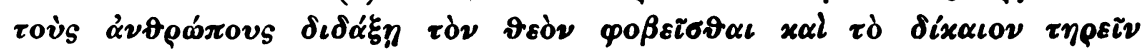

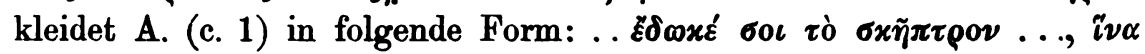

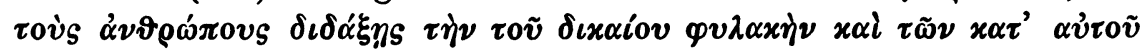

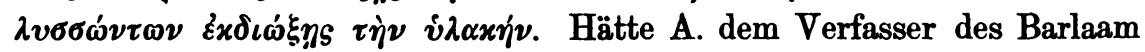
vorgelegen, so wäre dieser, vorausgesetzt, dafs er ändern wollte, durch

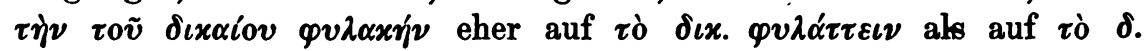

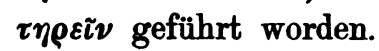

Der Gedanke $m$ wird im Roman mit diesen Worten abgeschlossen:

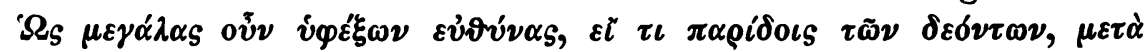

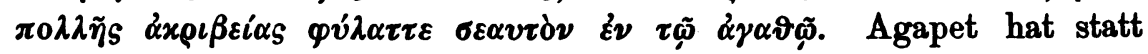

1) Die Wiener Handschrift hat $i \pi \varepsilon \rho \circ \psi i \alpha$ s. 


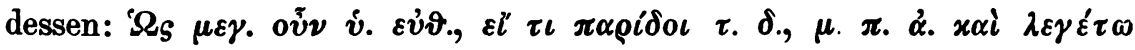
$\pi \alpha^{\prime} \nu \tau \alpha \quad x \alpha i \quad \pi \rho \alpha \tau \tau \varepsilon^{\prime} \tau \omega^{1}$ )

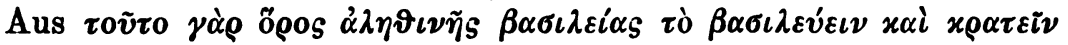

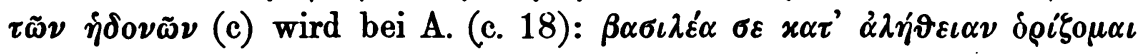

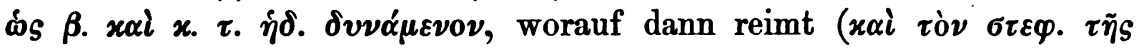

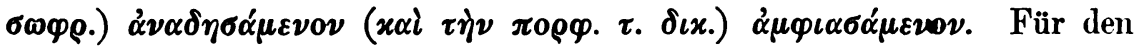
Autor des B. wäre die nächstliegende Umformung gewesen: $\beta \alpha \sigma \iota \lambda \varepsilon^{\prime} \alpha$

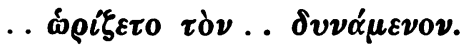

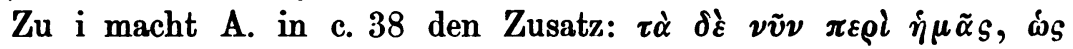

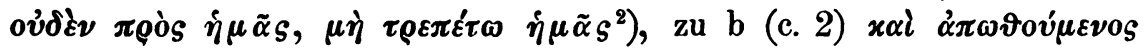

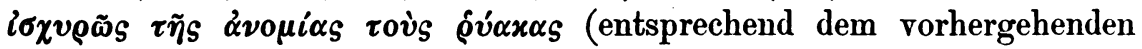

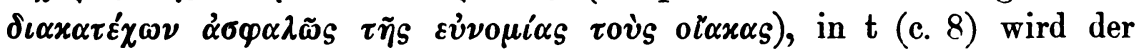

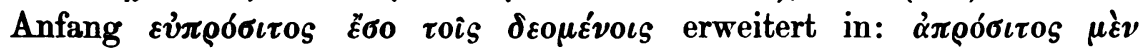

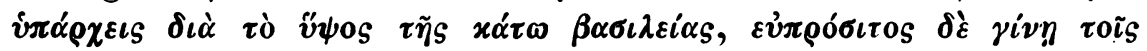

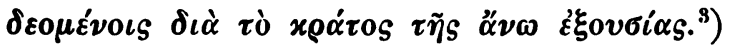

An zwei Stellen hat A. aus rhetorischen Gründen Sätze eingeschoben, die der Roman nicht kennt. In c. 37 folgen auf $\delta v ́ \nu \alpha u \iota \nu$ die

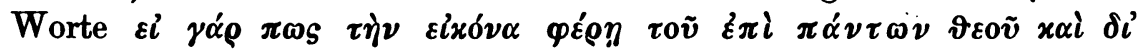

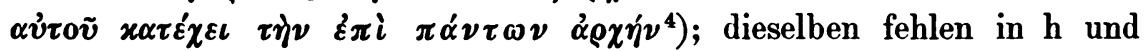
in q. Kap. $11(=n)$ enthält in den mir zugänglichen Ausgaben dieselben machen über hdschr. Lesarten keine Mitteilung - nach

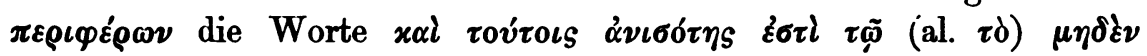

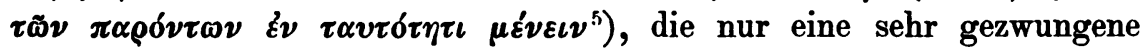
Erklärung zulassen. Man erwartet den Gedanken: das ist in der Ungleichheit das Gleiche, dals sie überall herrscht, und nichts von ihrem Gesetze ausgenommen ist. Diesen Gedanken bringt Greg. Naz. or. 17 p. 320 c, eine Stelle, die jedenfalls dem Verfasser vorgelegen hat, und die im Verein mit A.s rhetorischen Neigungen eine sichere Heilung der verderbten Worte ermöglicht. Gregor schreibt

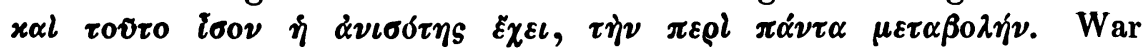

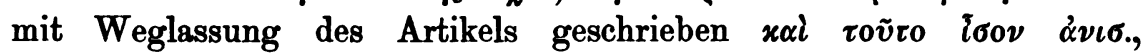

1) Auch hier stört A. durch diese Umformung den Zusammenhang. Jenes ต

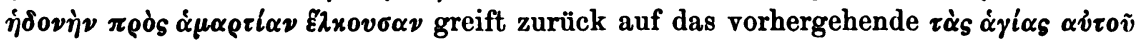

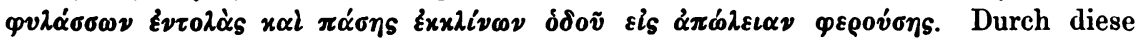
Zurückbeziehung erhält der ganze Abschnitt ein festes Gefüge.

2) Zu der Wiederholung des nämlichen Wortes vgl. das Beispiel aus Chorikios bei Seitz a. a. O. S. 45.

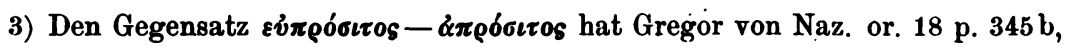
aber in anderm Zusammenhange.

4) S. d. vorletzte Anm.

5) So (und zwar $\tau \tilde{\omega}$ ) auch die Wiener Handschrift. 


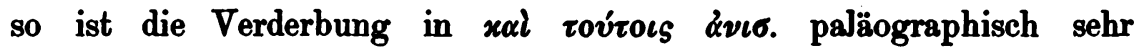

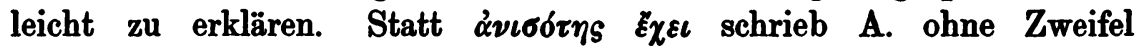

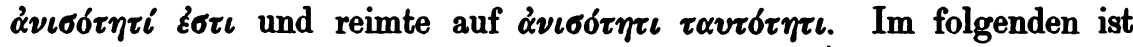
tò das Ursprüngliche. So verdankt auch dieses Einschiebsel A.s Vorliebe für rhetorische Floskeln seine Entstehung.

Endlich ist noch eines Falles zu gedenken, in welchem der Rhetor durch das Streben nach Sprachreinheit sich zu einer Umänderung des vorgefundenen Textes bewegen liefs. m stimmt abgesehen von der oben besprochenen Verschiedenheit im Schlufssatze und von Differenzen

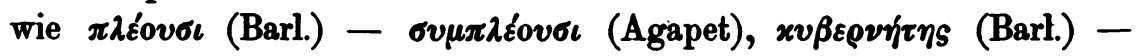
$\alpha$ ữos $\delta x v \beta$. (Ag.) mit c. 10 wörtlich überein, nur entspricht dem हैv

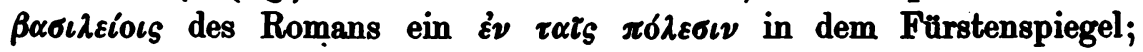
dementsprechend hat der erstere nachher $\beta \alpha \sigma \iota \lambda \varepsilon v ́ s$, der letztere ő $\rho \chi \omega \nu$. Hier ist wieder nicht zu verstehen, weshalb der Verfasser des Romans die Änderung vorgenommen haben sollte, hingegen springt in die Augen, dafs A. guten Grund hatte, das in der Bedeutung "Königreich“

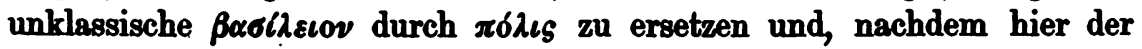
weitere Begriff an die Stelle des engeren getreten war, auch nachher

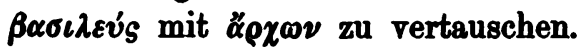

Wir sind in der Lage, auf das, was über die rhetorisierenden Umformungen Agapets gesagt wurde, die Probe zu machen. A. befindet sich in einer ebenso weit gehenden Übereinstimmung, wie mit den besprochenen beiden Abschnitten des Barlaamromanes, mit zahlreichen Stellen solcher Schriftwerke, über deren Priorität kein Zweifel besteht. Eine Prüfung ergiebt nun hier ganz den gleichen Sachrerhalt: bei engem Anschlufs an seine jedesmalige Vorlage stutzt A. dieselbe doch nach rhetorischen Rücksichten zu. Die folgende Zusammenstellung läist auch ohne jeden Kommentar dieses Verhältnis klar hervortreten.

Basil. in psalm. 1 p. 220 cf. Migne.

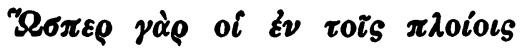

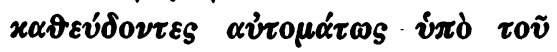

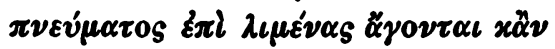
$\alpha$

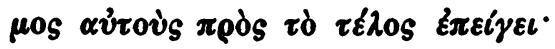

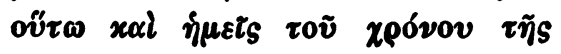

Agap. c. 70. ${ }^{1}$ )

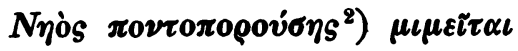

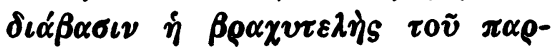

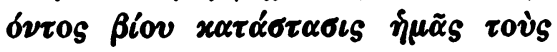
$\alpha \dot{v} \tau \tilde{\eta} S \quad \pi \lambda \omega \tau \tilde{\eta} \rho \alpha S$ $\lambda \alpha \nu \vartheta \alpha^{\prime} \nu 0 v \sigma \alpha x \alpha i$

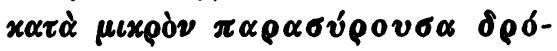

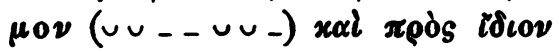

1) Der Anschlufs ist hier weniger eng als an den meisten der folgenden Stellen. Doch ist an einem Abhängigkeitsverhältnis wohl nicht zu zweifeln.

2) Vgl. Greg. Nazianz. or. 14 p. 270 a. 


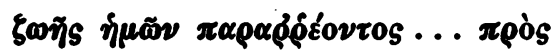

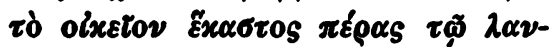

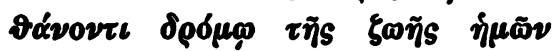

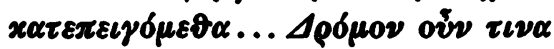

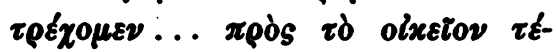
$\lambda$ LS $\varepsilon \pi \varepsilon \iota \gamma \delta \mu \varepsilon v 0 \iota$.

Gregor. Naz. or. 14 p. 271 c. der Paris. Ausg. v. 1840. ${ }^{1}$ )

$T i S \pi \alpha \rho \alpha \delta \rho \alpha \mu \varepsilon i \tau \alpha \iota \quad \tau \dot{\alpha} \pi \alpha \rho \alpha-$

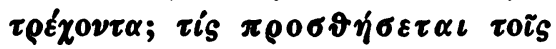

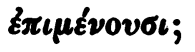

\section{Basil. in psalm. 61 p. $481 \mathrm{a} M$.}

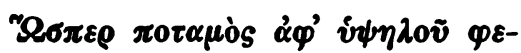

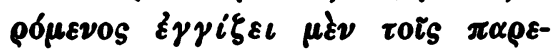

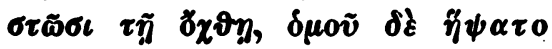

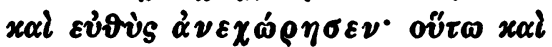

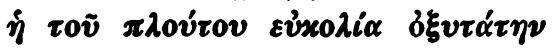

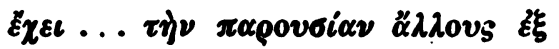

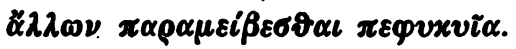

Basil. hom. in div. p. $296 \mathrm{c}$.

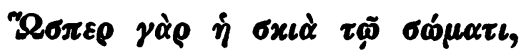

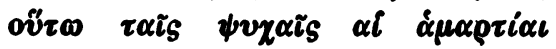

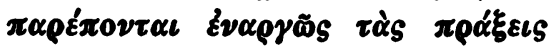

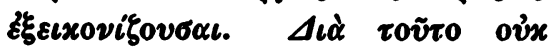

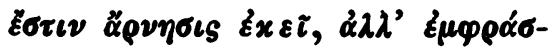

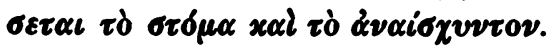

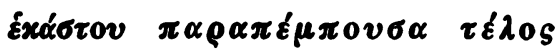
(

$\pi \alpha \rho \alpha \delta \rho \alpha \dot{\mu} \mu \omega \mu \varepsilon \nu \tau \dot{\nu} \pi \alpha \rho \alpha \tau \rho \varepsilon ́ \chi 0 \nu-$

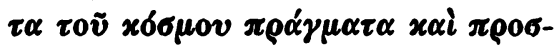

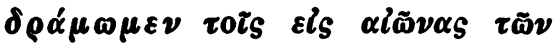

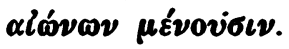

Agap. c. 7.2)

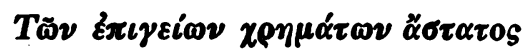

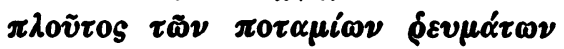

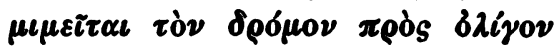

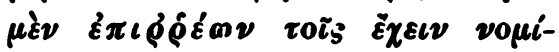

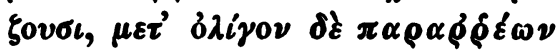

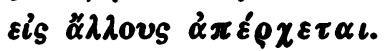

Agap. c. 69.

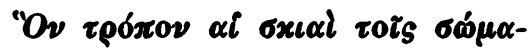

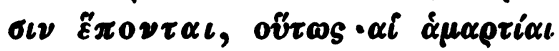

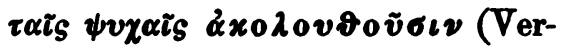
meidung des zusammengezogenen

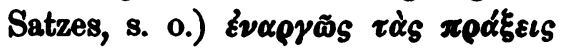

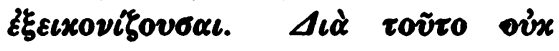

1) Die Stelle (von p. 270 a an) ist auch im Barlaamroman p. 127 und 218 benutzt. Wie der Anschlufs an den Wortlaut des Gregor hier enger ist, 80 hat auch die zweite Stelle einiges aus der Quelle, was A. völlig fremd ist. Doch lassen sich darauf keine Schlüsse bauen, da A. genugsam eigene Kenntnis des

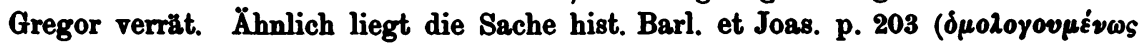

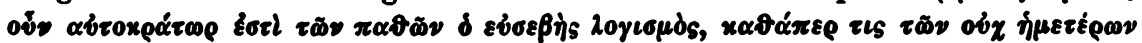

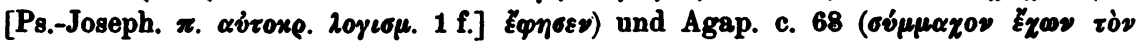

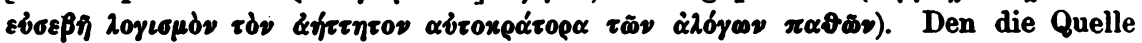
betreffenden Zusatz konnte der Verfasser d. B. nicht aus A. entnehmen. Andererseits traut man dem Zufall wohl nicht allzuviel zu, wenn man annimmt, dafs auch A. unabhăngig von B. (vielleicht durch ein Florilegium) auf die Stelle verfiel.

2) Ich kommè für diese Stelle auf die Quellenfrage unten noch zurück. 


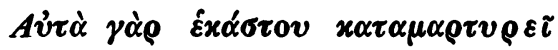

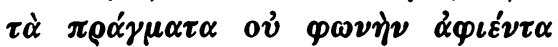

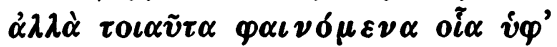
$\dot{\eta} \mu \tilde{\omega} \nu x \alpha \tau \varepsilon \sigma x \varepsilon \dot{v} \alpha \sigma \tau \alpha \iota$.

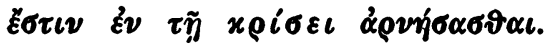

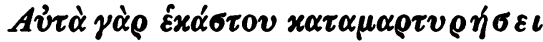

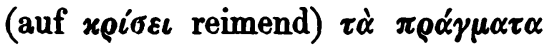

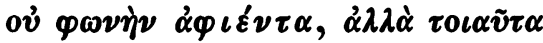
$\varphi \alpha \nu \varepsilon^{\prime} \nu \tau \alpha$ oi $\alpha \quad \pi \alpha \rho^{\prime} \quad \dot{\eta} \mu \tilde{\omega} \nu \quad \xi \pi \rho \alpha^{\prime}-$ $x \theta \eta \sigma \alpha \nu$ (wegen $\pi \rho \alpha \dot{\gamma} \gamma \mu \alpha \tau \alpha$ ).

Basil. in div. p. 292 cf.

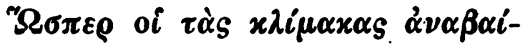

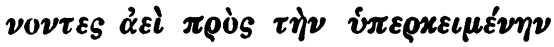

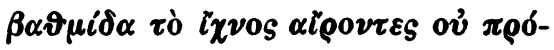

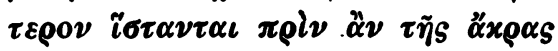

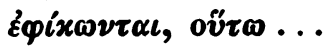

Gregor. Nazianz. or. 2 p. $19 \mathrm{c}$.

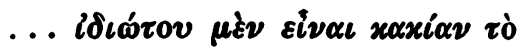

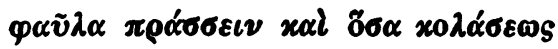

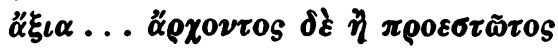

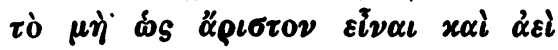

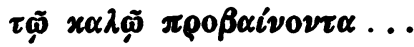

Greg. Nazianz. or. 14 p. $272 \mathrm{de}$.

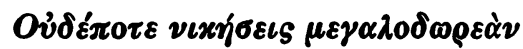

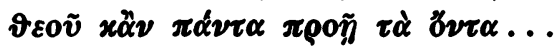

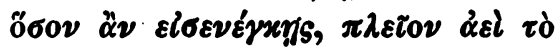

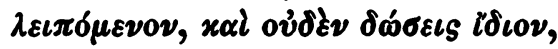

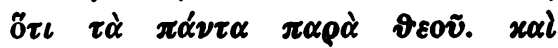
$\tilde{\omega} \sigma \pi \delta \rho$ ov่x $\varepsilon \sigma \tau \iota \nu$ i่ $\pi \varepsilon \rho \beta \tilde{\eta} \nu \alpha \iota$ $\tau \dot{\eta} \nu$

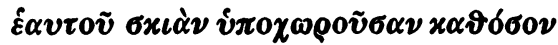

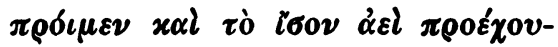

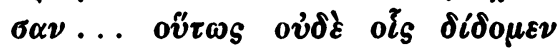

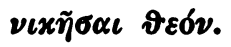

\section{Agap. c. 72.}

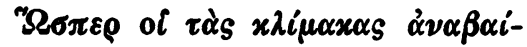

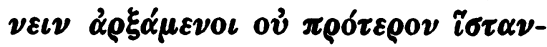

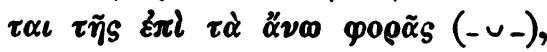

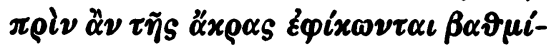
$\operatorname{\delta os}(-u \underline{)})$, ớ $\left.\tau \omega \ldots{ }^{1}\right)$

\section{Agap. c. 66.}

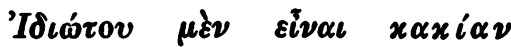

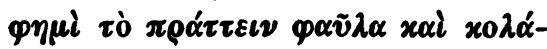

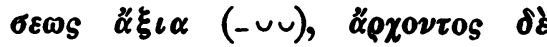

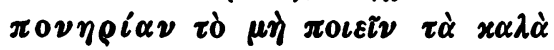

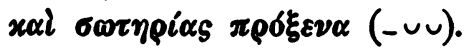

\section{Agap. c. 43.}

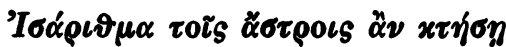

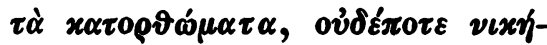

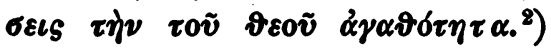

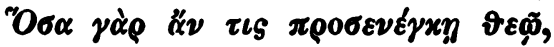

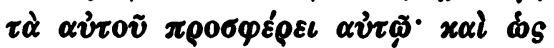
ov่u

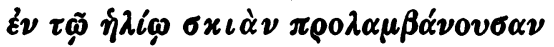

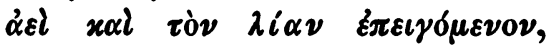

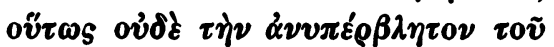

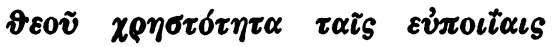
$\dot{v} \pi \varepsilon \rho \beta \eta \tilde{\eta} \sigma 0 \nu \tau \alpha \iota \stackrel{\alpha}{\nu \vartheta \rho \omega \pi 0 \iota}$.

1) Das zweite Glied des Vergleichs bilden bei Basil. die unersättlichen Reichen, bei Ag. der nach dem Edlen strebende König. Einen der Agapetstelle näher stehenden Vergleich hat Basileios homil. in psalm. 1 p. $217 \mathrm{cf}$. S. auch die von Greg. Naz. or. 14 p. 271 d angeführte Stelle Psalm. 83, 6, Greg. Naz. or. 2 p. 19b und Theodoros' Lobrede auf Theodosios S. 14 Usener. - Zum kretischen Kolonschlufs vgl. Seitz a. a. O. S. 43.

2) Vgl. Seitz a. a. O. S. 45.

Byzant. Zoitsohrift II 9 n. 4. 
Greg. Naz. ep. 21 (al. 107).

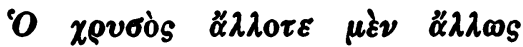
$\mu \varepsilon \tau \alpha \pi 0 \iota \varepsilon i \tau \alpha \iota$ xal $\sigma x \eta \mu \alpha \tau i \xi \varepsilon \tau \alpha \iota$ els

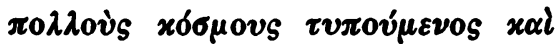

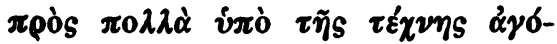

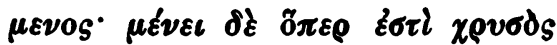

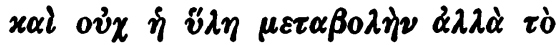
$\sigma \chi \tilde{\eta} \mu \alpha \lambda_{\alpha} \lambda \mu \beta \alpha^{\prime} \nu \varepsilon \iota$.
Agap. c. 34 .

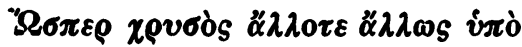
$\tau \tilde{\eta} s \tau \varepsilon \dot{x} \nu \eta S \mu \varepsilon \tau \alpha \tau v \pi 0 v \mu \varepsilon \nu 0 S x \alpha i$

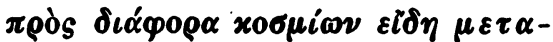

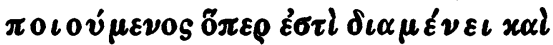

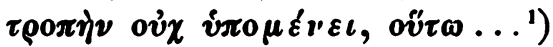

Wenn nun nach unserer bisherigen Untersuchung der Verfasser von B. u. J. nicht aus A. geschöpft haben kann, ist dann vielleicht das Verhältnis das umgekehrte und der Roman die Quelle des Fürstenspiegels? Ich glaube, auch diese Frage verneinen zu sollen. Darauf,

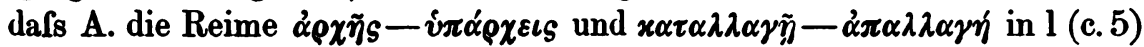
und $\nabla$ (c. 64), die ganz nach seinem Geschmacke sind, verschmäht,

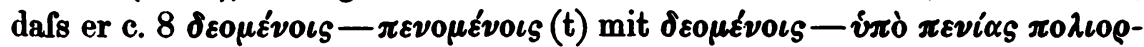

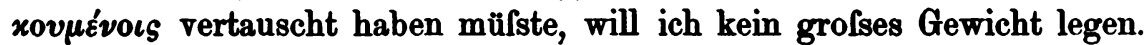
Vielleicht trieb ihn hier sein Rhetorenehrgeiz, die Kunststücke anderer durch solche eigener Mache, wenn sie auch bescheidener ausfielen, zu ersetzen, ein Zugeständnis, welches übrigens in A.s sonstigem Verhalten wenig Unterstützung findet. Entscheidend ist, dafs A. an einer Stelle die Quelle vollständiger wiedergiebt als der Roman und an einer andern Stelle ihren Wortlaut genauer erhalten hat. Ersteres ist der Fall in c. 7 (vgl. mit $f$ und der oben ausgeschriebenen Stelle Basil. in psalm. 61 p. $481 \mathrm{a})$. Die den Worten $\varepsilon \gamma \gamma i \xi \varepsilon \iota-\not \partial \psi \alpha \tau 0-\alpha \dot{\alpha} \varepsilon \chi \dot{\omega} \varrho \eta \sigma \varepsilon \nu$

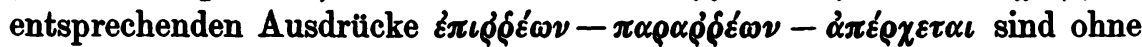

1) Ein weniger sicheres Abhängigkeitsverhaltnis ergiebt die Vergleichung

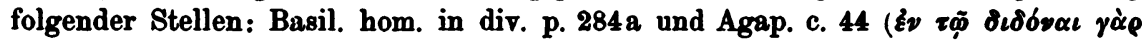

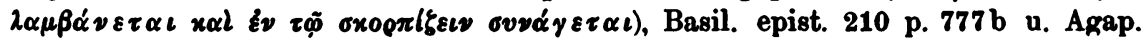
c. $9\left(x \alpha \tau \alpha \sigma \tau \rho \alpha^{\prime} \pi \tau \eta \tau \alpha \iota-\delta \iota \alpha^{\prime} \sigma \times \eta \tau \alpha \iota\right)$; zum Gedanken vgl. auch Greg. Naz. or. 2 p. 11d, Theodoros' Lobr. auf Theodos. S. 87 Usener.

Von sonstigen Parallelen zu Agapet bei Basileios und Gregor führe ich noch

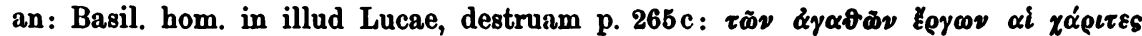

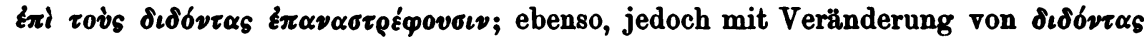

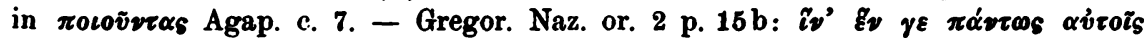

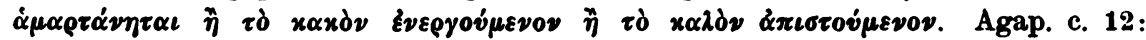

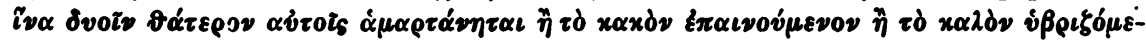

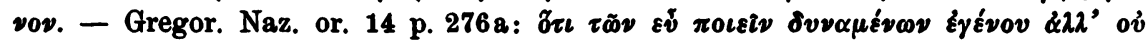

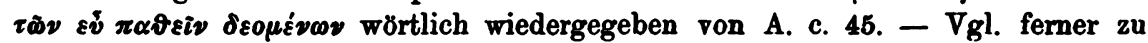
Agap. c. 10 Gregor. Nazianz. or. 17 p. 322 d, zu c. 50 Gr. Naz. or. 14 p. 278 b, zu c. 23 Greg. Naz. or. 14 p. 284 c ( or. 14 p. 258 d u. 261 b, zu c. 28 Basil. regul. brev. tract. p. 1112 cf. Migne, zu c. 54 Greg. Naz. or. 3 p. 71 c, zu c. 16 (Basil.) de virt. et vit. p. 1120 c. 
Parallele im Barlaam. Hat nun hier weder der Verfasser des Romans aus A., noch dieser aus dem Roman geschöpft, so bleibt nur die Annahme übrig, dals beide auf eine gemeinsame Quelle zurückgehen, in welcher der Anfang der Basileiosstelle die Gestalt erhalten hatte, welche uns nun in beiden Werken vorliegt, darauf aber auch der Rest der Basileiosstelle wiedergegeben wurde, den nur A. sich zu eigen machte. Die zweite Stelle ist Ag. c. 11 Anf;; sie geht zurück auf Greg. Naz. or. 14 p. 270 a (vgl. auch or. 17 p. 319 e, epist. 29 [al. 18]): xúxגos

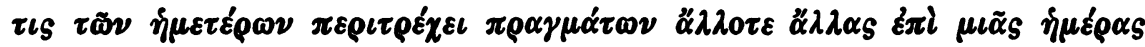

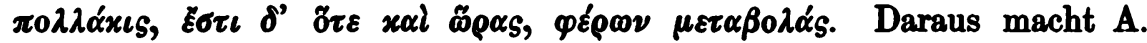

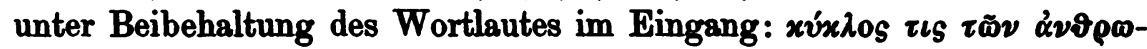

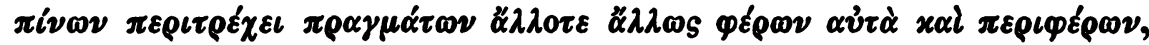
während der Verfasser der Mönchserzählung mit Veränderung des Ein-

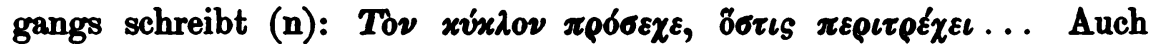
hier werden wir wieder auf die Annahme einer gemeinsamen Vorlage geführt, an deren Text A. festgehalten hätte, während der Verfasser des B. ihn in der angegebenen Weise umgestaltete. Eine Möglichkeit wäre noch vorhanden, um an beiden Stellen dieser Folgerung zu ent-

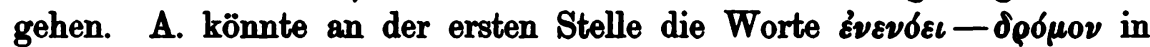
dem Romane vorgefunden und dieselben selbst mit der Basileiosstelle kontaminiert und ebenso an der zweiten Stelle die Worte des Barlaam der ihm im Original bekannten Gregorstelle wieder näher gebracht haben. Man wird aber um so weniger geneigt sein, zu diesem Auskunftsmittel zu greifen, als ein weiteres Moment die Annahme einer gemeinsamen Quelle sehr unterstützt: Der erste der beiden in Betracht kommenden Abschnitte des B. lälst an deutlichen Spuren die Umarbeitung einer Vorlage erkennen. Die Wendungen

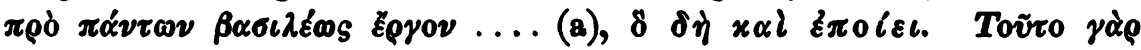

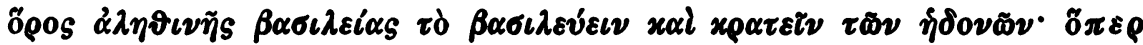

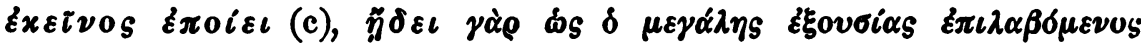

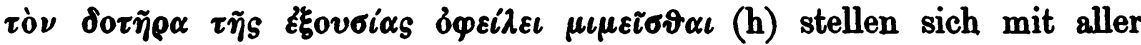
Deutlichkeit als Mittel dar, einen Befehl in Erzählung umzuformen. Die Vorlage enthielt Gebote, hier verlangte der Zusammenhang, dafs von Joasaph die Befolgung dieser Gebote ausgesagt werde. Die Übertragung geschah in dieser etwas schwerfälligen Weise, für welche übrigens vielleicht die Absicht, den bekannten Wortlaut eines Originals möglichst wenig zu verwischen, einen Entschuldigungsgrund bildet.

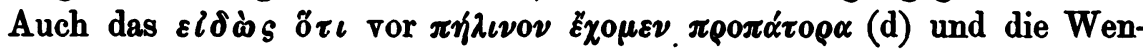

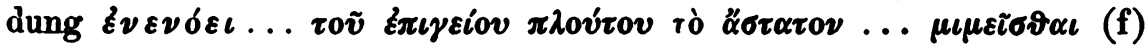
dienen dazu, eine in der Vorlage im Präsens ausgesprochene Wahrheit in den Zusammenhang der Erzählung einzuordnen. Die an das pro- 


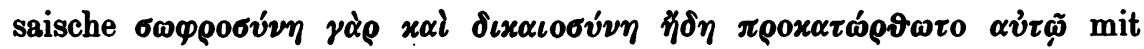

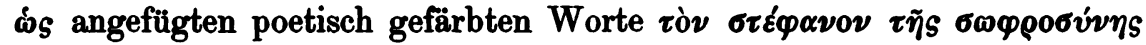

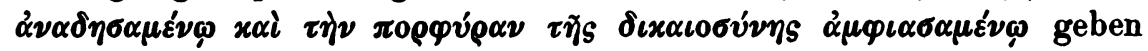
sich als Zitat.

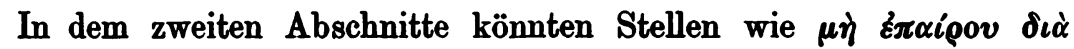

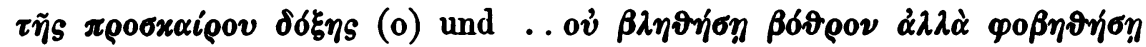

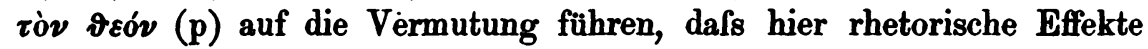
einer Quelle nicht beachtet und daher verwischt wurden; der Reim

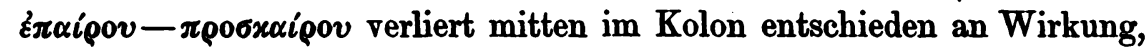

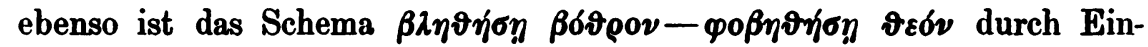
fügung des Artikels vor $\vartheta \varepsilon \delta \nu$ zerstört. (Dafs die gemeinsame Quelle rhetorischem Aufputz nicht abgeneigt war, zeigen ja auch die im B. u. Agapet übereinstimmenden Partien mehrfach.) Doch käme selbstverständlich solchen Spuren dann erst Bedeutung zu, wenn sie durch eine weitere Untersuchung sehr vermehrt werden könnten.

Vielleicht gelingt es Mitforschern, welchen eine bessere Kenntnis der patristischen Litteratur zu Gebote steht, den Faden, der auf jene Quelle führt, weiter aufzudecken, oder, wenn sich meine Annahme einer gemeinsamen Vorlage als falsch erweisen sollte, doch sonst neues Material zur Lösung dieser wichtigen Frage beizusteuern.

Bern.

Karl Praechter. 